\title{
Mulberry fruit as an antioxidant component in muesli
}

\author{
Joanna Kobus-Cisowska, Anna Gramza-Michalowska, Dominik Kmiecik, Ewa Flaczyk, \\ Józef Korczak
}

Faculty of Food Science and Nutrition, Poznan University of Life Sciences, 31 Wojska Polskiego Str., 60-624 Poznan, Poland;

${ }^{*}$ Corresponding Author: joannak@up.poznan.pl

Received 2013

\begin{abstract}
The aim of the study was to develop the technology of muesli via analyzing the addition of white mulberry fruit. The first step involved the selection of ingredients from bioactive compounds. Next, the technology of production was developed. Then, the antioxidant activity of new muesli with potentially pro-health model systems was estimated. Muesli was characterized in terms of its basic nutritional value, and antioxidant potential. The ability to inactivate DPPH and $A B T S$ radicals was estimated. In addition, the amount of fat in muesli was calculated. As part of this calculation, fatty acid composition and content of primary and secondary oxidation products were ascertained. The last stage of the project included sensory analysis of the resultant products. Our studies showed that the new product-muesli had high nutritional value, high antioxidant potential and a positive fatty acid composition. And sensory analysis showed that the product was attractive.
\end{abstract}

Keywords: Mulberry; Morus Alba; Muesli; Antioxidant Properties; Bioactive Food

\section{INTRODUCTION}

There is an increasing awareness of diseases associated with diet. Numerous analyses have shown that foods containing phytochemicals with antioxidant compounds have a strong protective effect against major diseases; for example cardiovascular diseases, diabetes, cancer, obesity and many others [1-3]. Most antioxidant compounds in food are derived from different plants and belong to various classes of compounds with a wide variety of physical and chemical properties - carotenoids, dietary glutathione polyphenols, and vitamins. Moreover, these compounds also play a significant role in the assurance of high quality food, including mainly fat containing food, by the inhibition of oxidative changes to this fat. Lipid oxidation is a highly deteriorative process in foods, which lead first of all to unacceptable properties for the customer and most importantly a loss in nutritional value [4-7].

The mechanism of polyphenol activity is highly differentiated. They might act as reducing substances, compounds blocking free radicals, chelating metal ions, catalysts of oxidation reaction, inhibitors of the activity of enzymes contributing to free radical creation, e.g. xanthine oxidase, lipoxygenases or protein kinase. In addition, they may reduce reactive oxygen species to more stable forms [7-9].

The most commonly grown species in the Morus genus are white mulberry, black mulberry, and red mulberry $[10,11]$. Mulberries (Morus alba) have long been used in traditional medicine to improve eyesight, lower blood pressure, prevent diabetes, protect the liver, strengthen joint and treat fever. Fruits, leaves, sprouts as well as bark are currently used in production processes, and various forms of final products, including syrups, jams, ice-cream, vinegar or alcohol, are produced from them $[11,12]$. As may be concluded from numerous reports, the fruits of the white mulberry (Morus alba L.), due to their characteristic composition and properties, may additionally profitably affect the human organism. Mulberry fruits are rich in organic acids such as malic acid, citric acid, and tartaric acid [3,10,12]. This fruit contains essential fatty acids, as well as vitamins and polyphenols which are effective antioxidants [11].

The present study aimed to develop a technology for producing muesli with the fruit of the white mulberry.

This object was achieved by:

- Selection of materials containing potentially bioactive compounds,

- Development of production technology by detailing the percentage of each fraction,

- Evaluation of the antioxidant activity of the resulting muesli with potentially pro-health model systems.

\section{MATERIALS AND METHODS}

\subsection{Material}

Ingredients used for muesli preparation were purchased 
in local stores, with a minimum three months of shelf- life. These included: oatmeal (Kupiec Sp. z o.o., Krzymów, Poland), linseed (Sante, Warsaw, Poland), granulated oat bran (Sante, Warsaw, Poland), dried appled (Paula, Kalisz, Poland), dried cranberry (Bakalland, Warsaw, Poland), dried mulberry fruits (Bio Planet, Leszno, Poland).

All solvents were of analytical grade unless otherwise specified. 2,2-Diphenyl-1-picrylhydrazyl. (DPPH.), 6hydroxy-2,5,7,8-tetramethylchroman-2-carboxylic acid (Trolox), and Folin-Ciocalteau reagent were purchased from Sigma-Aldrich (St. Louis, MO, USA). The other chemicals were obtained from Merck (Darmstadt, Germany) and POCH (Gliwice, Poland).

\subsection{Muesli Preparation}

A base muesli formulation, taken from the literature [9] and slightly modified was used as a reference and was prepared in the laboratory (sample A). Muesli samples (with apple and cranberry - sample B and with an addition of apple, cranberry and mulberry fruit-sample C) were prepared according to Table 1.

\subsection{Chemical Characteristics of Muesli: Protein, Total Dry Matter, Lipids, Ash, Fatty Acid Composition}

All analyses were performed on these 3 samples in 3 replications according to method AOAC [13]. The moisture of samples was determined by drying at $103 \pm 2{ }^{\circ} \mathrm{C}$ until they reached a constant weight. Protein content was determined by the Kjejdal method $(\mathrm{N} \times 6.25)$, in Kjeltec 2200 (Foss Tecator, Sweden). Fat content was determined using the method of multiple constant extraction with petroleum ether, which was next evaporated, and the weighing method was used in the fat content examination. A Soxtec HT6 apparatus manufactured by Foss Tecator (Sweden) was used for the extraction. Ash content was examined by incineration in a muffle furnace Naberterm S 27- at a temperature of $535^{\circ} \mathrm{C}$, with air access.

Table 1. Composition of muesli flakes.

\begin{tabular}{lccc}
\hline \multicolumn{1}{c}{ Ingredients: } & sample A: & sample B: & sample C: \\
\hline oatmeal & $300 \mathrm{~g}$ & $300 \mathrm{~g}$ & $300 \mathrm{~g}$ \\
oat bran (granulated) & $90 \mathrm{~g}$ & $90 \mathrm{~g}$ & $90 \mathrm{~g}$ \\
linseed & $60 \mathrm{~g}$ & $60 \mathrm{~g}$ & $60 \mathrm{~g}$ \\
dried cranberry & - & $60 \mathrm{~g}$ & $60 \mathrm{~g}$ \\
dried apple & - & $60 \mathrm{~g}$ & $60 \mathrm{~g}$ \\
dried mulberry & - & - & $110 \mathrm{~g}$ \\
\hline
\end{tabular}

The percentage composition of fatty acids was determined using a gas chromatograph with an FID detector (Agilent Technologies 7820A GC) according to method described in PN-EN ISO [14]. The method involved dissolution of a fat sample with hexane, and its further trans-esterification using sodium methanol solution.

\subsection{Antioxidant Activity}

The of total phenolic content (TPC) in muesli was determined by visible spectrophotometry based on a colorimetric oxidation/reduction reaction according to Cheung et al. [15]. Ferulic acid was the standard employed in this work. The absorbance of the sample was measured at $\lambda=$ $725 \mathrm{~nm}$ using a SPECORD $\AA 40$ (Analytik Jena AG, Germany). Based on the construction of a standard curve, TPC data for the preparations from muesli were expressed as mg ferulic acid equivalents/g dry extract.

The free-radical-scavenging potentials of muesli were tested in a methanolic (80\%) and water solution of DPPH as described by Amarowicz et al. [16]. The extent of discoloration of the solution indicated the scavenging efficacy of the added substance. Absorbance was measured at $\lambda=517 \mathrm{~nm}$ (SPECORD $\AA 40$, Analytik Jena).

The ability of ABTS radical cation deactivation was examined according to the method described by Re et al. [17]. The examination involved determination of the degree of scavenging of ABTS + radical cations formed from ABTS by oxidation with potassium persulfate. The scavenging was measured spectrophotometrically at a wave length of $734 \mathrm{~nm}$ (SPECORD ${ }^{\circledR}$ 40, Analytik Jena, Germany). Antioxidant ability with ABTS radical cation application was calculated per Trolox for a standard curve, and the result was given as $\mathrm{mg}$ of Trolox $/ 1 \mathrm{~g} \mathrm{d.m}$. of an extract.

\subsection{Oxidative Stability Determination}

\section{Lipid extraction}

The process was run with the Folch mixture (chloroform: methanol 2:1 v/v). After the separation phase, chloroform was evaporated in a vacuum evaporator and the lipid fraction was obtained [18].

\section{Peroxide value}

The peroxide value (PV) was determined by titration with $0.02 \mathrm{M}$ sodium thiosulfate and was expressed in meq $\mathrm{O}_{2} / \mathrm{kg}$ [19].

\section{Anisidine value}

The determination was performed based on PN-ISO [20]. The method is based on the reaction of aldehydes present in the sample with a p-anisidine solution in ice-cold acetic acid and on the spectrophotometric absorbance measurement of the formed yellow complex at a wave length of $350 \mathrm{~nm}$ (Metertek SP-830, Taiwan). 


\subsection{Sensory Analysis}

The descriptive panel comprised 20 assessors (14 female and 6 male, aged 22 to $54 \mathrm{y}$ ), who were students or staff of the university.

Sensory assessment of the experimental samples of oat muesli was conducted in the sensory laboratory fulfilling the requirements provided in standard PN-ISO, [21].The method of descriptive quantitative analysis, i.e. sensory profiling, was used in the study. Qualitative determinants of color, smell, taste and consistency were evaluated. The intensity of each quality score was determined using a $10 \mathrm{~cm}$ structured linear scale with suitable margin denotations. The color (beige, brown, red, orange, green, purple) was evaluated on a scale from slightly intense to intense. Marginal designations, i.e. imperceptible - intense, were used for smell (fruit, cereal, foreign) and taste descriptors (sweet, salty, bitter, cloying, fruity, vegetable, and foreign). Marginal designations: large, small, were used for consistence descriptors (compactness, degree of steeping), while crunchiness was assessed on a soft-hard scale. Also, a general assessment of the product was performed. The results obtained were replaced with numerical values expressed in points.

\subsection{Statistical Analysis}

All analyses were made in at least three replications. Average and standard deviation values were calculated with the use of Microsoft Office Excel 2007 software. Pearson's correlation indexes were calculated with the use of STATISTICA ${ }^{\mathrm{TM}}$ PL 7.0 StatSoft. The significance of differences between mean values was determined at $p$ $\leq 0.05$ applying the analysis of variance (ANOVA) followed by Tukey's multiple range test.

\section{RESULTS}

\subsection{Chemical Characteristics of Muesli}

The chemical characteristics of muesli are given in Table 2.

The total protein content of muesli was between 12.05 (sample C with mulberry) and 16.43 (sample A - control), respectively. Control muesli (sample A), which had the lowest moisture content, had the highest total fat (9.81 $\%$ ), followed by sample B with apple and cranberry (8.53) and sample $\mathrm{C}$ with mulberry (6.93). The ash content of the examined muesli was between $1.20 \%$ (sample A control) and 1.60 (sample $\mathrm{C}$ with mulberry), respectively.

A similar chemical composition for muesli was presented by Pernille at al. researches [4,5], where protein and fat content was in a range up to $9 \%$ and $14 \%$, respectively. Also, the humidity of muesli in the study of the above-mentioned authors was the same as in the presented experiment, i.e. at a level of $4 \%-7 \%$.
Fatty acid analysis showed that the muesli studied contained six major fatty acids. Oleic acid, C18:1, was the dominant fatty acid $(34.68 \%-36.31 \%)$ in all muesli samples, followed by linoleic acid C18:2 (28.51\% 29.97\%). Our results are in agreement with Pernille et al [4], who reported that the fatty acids found at the highest levels of muesli were oleic acid (around 48\% - 49\%) and linoleic acid $(20 \%-31 \%)$. The examined muesli in the study by Pernille et al. [5] also contained the highest amount of oleic acid and linoleic acid, while stearic acid and linolenic acid were noted at the level of $2 \%-3 \%$ and $1 \%-5 \%$, respectively.

\subsection{Antioxidant Activity}

Antiradical activity towards ABTS and DPPH is presented in Figures 1 and 2.

Table 2. Chemical composition of muesli.

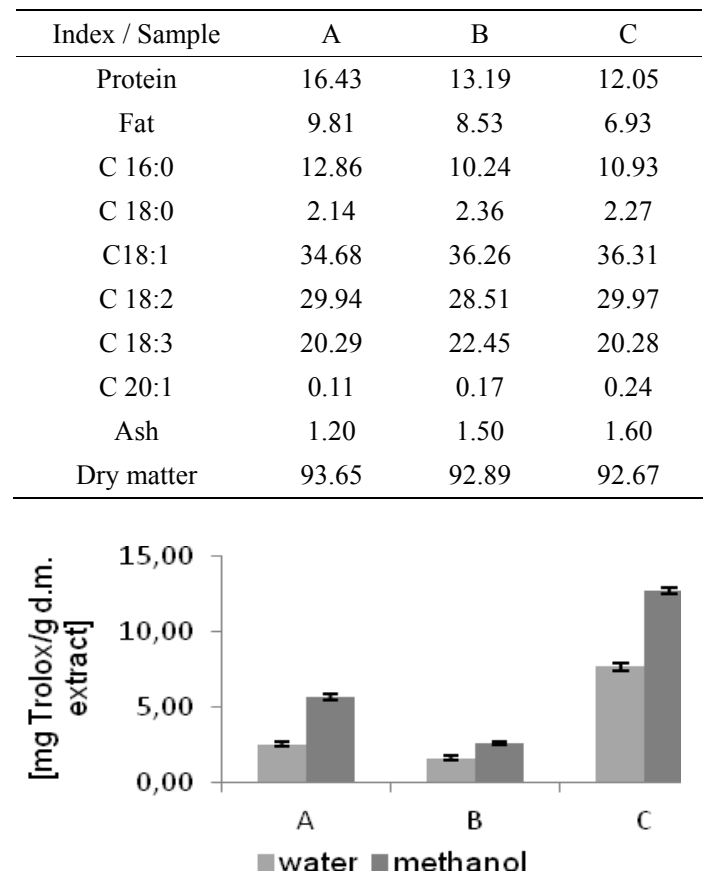

Figure 1. Antiradical potential of muesli extract measured with ABTS radicals.

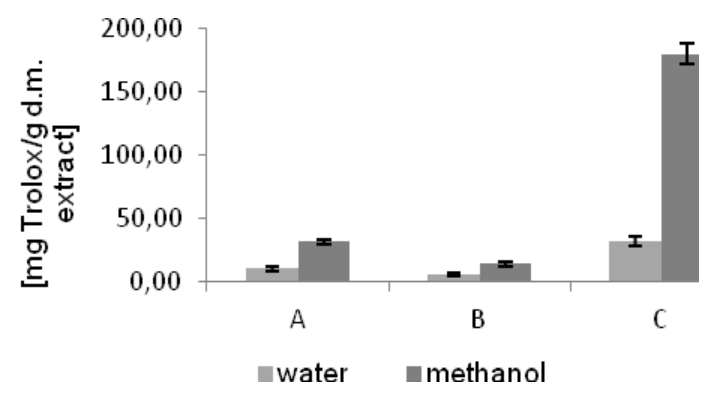

Figure 2. Antiradical potential of muesli extract measured with DPPH radicals. 
The muesli sample with added mulberry fruit was characterized by the highest activity towards ABTS: 7.71 $\mathrm{mg}$ Trolox/g d.m. for water extract and $12.73 \mathrm{mg}$ Trolox/ $\mathrm{g}$ d.m. for methanolic extract. Lower activity was noted in the control sample where the values were $2.55 \mathrm{mg}$ Trolox/g d.m. for water extract, and $5.71 \mathrm{mg}$ Trolox $/ \mathrm{g}$ d.m. for methanolic extract. In turn, the lowest activity towards ABTS radical cation was demonstrated for the sample with added cranberry and apple: $1.64 \mathrm{mg}$ Trolox/ $\mathrm{g}$ d.m. for water extract, and $2.62 \mathrm{mg}$ Trolox/g d.m. for methanolic extract, respectively.

Similar results were obtained in the test with DPPH. The highest values for antiradical activity were noted for muesli with white mulberry. Methanolic extract demonstrated a scavenging ability value of - $180.14 \mathrm{mM}$ Trolox/g d.m. of an extract, a 6-fold lower value for this sample was noted in the case of water extract $(31.70 \mathrm{mM}$ Trolox/g d.m. of an extract). The other two samples were characterized by considerably lower values. Antiradical activity for the control sample was at a level of $9.71 \mathrm{mM}$ Trolox/g d.m. for water extract, and $31.57 \mathrm{mM}$ Trolox $/ \mathrm{g}$ d.m. for methanolic extract. In turn, for the sample with added apples and cranberry it was $5.71 \mathrm{mM}$ Trolox/g d.m. for water extract, and $13.72 \mathrm{mM}$ Trolox/g d.m. for methanolic extract. Fruit addition, mainly mulberry, affected the antiradical activity of muesli. Numerous papers demonstrating the beneficial antioxidative effect of mulberry fruits are available in the literature. Chon et al. [11] demonstrated that ethanolic extracts of mulberry fruits scavenge radicals to the highest degree.

The total content of polyphenols is presented in Figure 3. The highest level of total polyphenols was noted in the sample of muesli with added mulberry fruit, in water extract, and then in methanolic extract: the level of polyphenols was 5.65 and $3.29 \mathrm{mg}$ of ferulic acid/g d.m. of an extract, respectively. In turn, the muesli sample with added apple and cranberry contained $1.71 \mathrm{mg}$ of ferulic acid $/ \mathrm{g}$ d.m. for water extract, and $0.55 \mathrm{mg}$ of ferulic acid/g d.m. for methanolic extract.

An increase in polyphenol levels in muesli is explained by the increase in the amount of fruits which are a source of these components. It was demonstrated in the

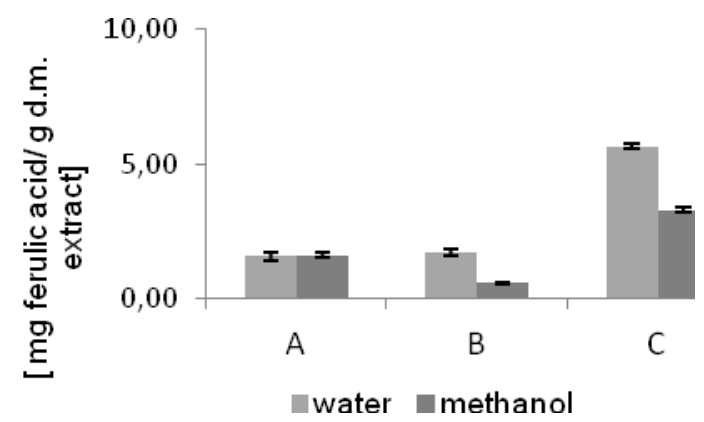

Figure 3. Polyphenolic content in muesli extract. study by Ercisli and Orhan [10] that white mulberry fruits contain as much as $181 \mathrm{mg}$ in fresh mass. Similar results comparing polyphenol content in various anatomical parts of white mulberry were presented by Chon et al. [11], who noted total polyphenol content in mulberry fruits at a level of $213 \mathrm{mg} / \mathrm{kg}$. As may be concluded from the study by Bae et al. (2007) mulberry fruit extracts contained as much as $2570 \mu \mathrm{g} / \mathrm{g}$ of polyphenols expressed as gallic acid. The relationship between polyphenolic compound content and antiradical activity was also confirmed in this study. As demonstrated by Gundogdu et al. [12] and Pawlowska et al. [23] rutin, chlorogenic, gallic and caffeic acids are the predominant polyphenols in white mulberry fruits.

\subsection{Oxidative Stability Determination}

Muesli is susceptible to lipid oxidation due to the high content of unsaturated fatty acids. The oxidative processes also depend on the content of compounds which may theoretically inhibit these unprofitable changes. Oxidative stability was determined in the study by examination of the content of primary and secondary oxidation products, and the results are presented in Table 3.

The content of primary oxidation products was in the range of $5.78 \mathrm{meq} \mathrm{O}_{2} / \mathrm{kg}$ for the control sample to 9.47 meq $\mathrm{O}_{2} / \mathrm{kg}$ for the sample containing mulberry fruits. The content of secondary oxidation products expressed as an anisidine number was in turn nearly two-fold lower in sample $\mathrm{C}$ (18.01) when compared to the reference sample A (33.43). Various contents of primary and secondary oxidation products result probably from the different compositions of the examined muesli. Moreover, as may be concluded from the study by Bae et al. [22], active compounds of mulberry fruits considerably moderate fat oxidation. It was demonstrated in model tests that formation of secondary oxidation products was as much as $23 \%$ higher.

\subsection{Sensory Analysis}

The results of sensory assessment are presented in Figure 4. Beige color was predominant characteristic for all samples. Purple and red colors were also noted in the sample with apple and cranberry, while orange color was additionally noticed in the sample with mulberry.

Table 3. Oxidative stability of muesli recorded by peroxide and anizidine value.

\begin{tabular}{ccccccc}
\hline & \multicolumn{3}{c}{ Peroxide value $\left[\mathrm{meq} \mathrm{O}_{2} / \mathrm{kg}\right]$} & \multicolumn{3}{c}{ Anizidine value } \\
\hline Muesli A & 5.78 & \pm & 0.76 & 33.43 & \pm & 1.44 \\
Muesli B & 5.92 & \pm & 0.28 & 34.85 & \pm & 1.95 \\
Muesli C & 9.47 & \pm & 0.47 & 18.01 & \pm & 0.98 \\
\hline
\end{tabular}




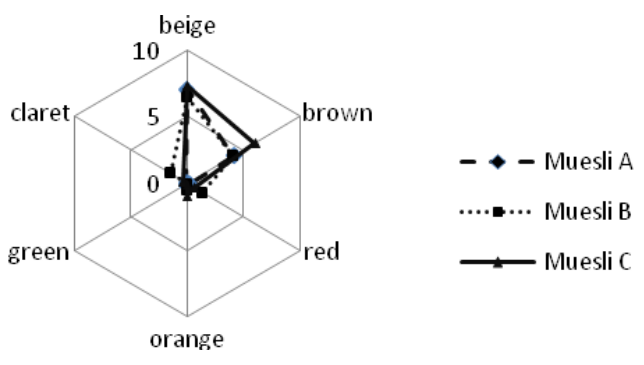

(a) colour of muesli

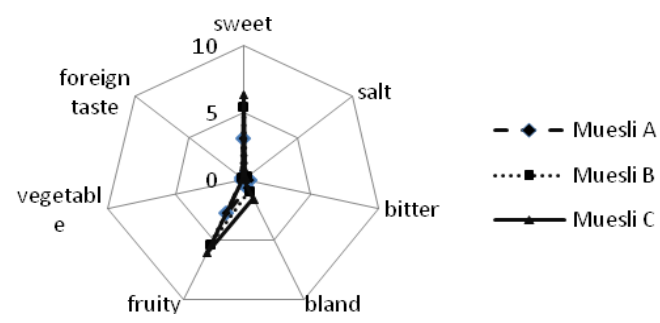

(b) taste of muesli

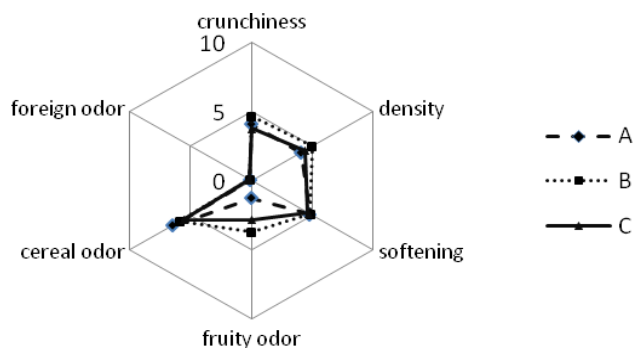

(c) odor of muesli, crunchiness, density and softening

Figure 4. Sensory analysis of muesli (a) colour of muesli;(b)taste of muesli;(c)odor of muesli and crunchiness, density, softening).

Cereal smell was predominant in all samples. Further, a fruity smell was also perceived, which was especially distinct in the sample with added apple and cranberry, and mainly in the sample with added white mulberry.

Sweet and fruity taste was especially highly evaluated in the examined samples. Also, a subtly cloying taste was noticed, and even bitter to a small degree in the control sample without fruits. Comparable crunchiness evaluation results were obtained in the examination, however the highest crunchiness was noted for muesli with white mulberry -4.66 points, then for the control sample 4.09 points, and muesli with apple and cranberry 3.81points (on a 1 - 10 scale, where 1 means soft muesli flakes, and 10 - hard ones). People tend to like contrasts in foods, and products which undergo changes in texture during mastication are well liked [6]. As may be concluded from these authors' research, the choice of muesli is mainly affected by a sweet taste, which may be derived from dried fruits. It can be noticed in the results of this study that sweet taste was to the highest degree detectable in the sample of muesli $\mathrm{C}$ with added mulberry fruits.

\section{CONCLUSIONS}

Muesli including oat flakes, linseed, granulated bran, cranberry, apple and white mulberry fruit was characterized by high nutritional value. The content of saturated acids in muesli was at a level of about $13 \%$, monounsaturated at a level of $37 \%$, and polyunsaturated $50 \%$. Oleic acid was found in the highest amount, and linoleic acid was predominant among polyunsaturated ones. The study demonstrated less advanced oxidative changes of the product with added white mulberry fruit. Moreover, the examined samples exhibited antioxidative properties measured in free radical tests with ABTS and DPPH, which resulted from a higher content of polyphenolic compounds also originating from dried fruits. The conducted sensory analysis allowed us to notice that an addition of mulberry fruits profitably influenced the sensory attractiveness of the product.

Thus, mulberry fruits may be an attractive component of muesli due to an increase in antioxidative activity, as well as profitable sensory properties and nutritional value. Muesli based on oat flakes, granulated bran and linseed with an addition of white mulberry fruits may constitute an important element of a diet.

\section{ACKNOWLEDGEMENTS}

Financially supported by EU Project Nr POIG 01.01.02-00-061/0.

\section{REFERENCES}

[1] Andallu, B. and Varadacharyulu N. Ch. (2003) Antioxidant role of mulberry (Morus indica L. cv. Anantha) leaves in streptozotocin-diabetic rats. Clinica Chimica Acta, 338, 1-2, 3-10. doi:10.1016/S0009-8981(03)00322-X

[2] Andallu, B. Radhika and Suryakantham,V. (2003) Effect of aswagandha, ginger and mulberry on hyperglycemia and hyperlipidemia, Plant Foods for Human Nutrition, 58, 1-7. doi:10.1023/B:QUAL.0000040352.23559.04

[3] Murata, K., Yatsunami, K. Fukuda, E. (2004) Antihyper-glycemic effects of propolis mixed with mulberry leaf extract on patients with type 2 diabetes, Alternative Therapies in Health and Medicine, 10, 78-79.

doi:10.1016/j.foodchem.2004.05.043

[4] Jensen, P. N., Danielsen, B., Bertelsen, G., Skibsted L. H. and Andersen M. L. (2005) Storage stabilities of pork scratchings, peanuts, oatmeal and muesli: Comparison of ESR spectroscopy, headspace-GC and sensory evaluation for detection of oxidation in dry foods. Food Chemistry, 91, 25-38.

[5] Jensen, P. N., Christensen J. and Engelsen S. B. (2004) Oxidative changes in pork scratchings, peanuts, oatmeal and muesli viewed by fluorescence, near-infrared and infrared spectroscopy. European Food Research and Technology, 219, 294-304. doi:10.1007/s00217-004-0954-x 
[6] Kalviainen, N., Salovaara, H. and Tuorila H. (2002) Sensory attributes and preference papping of muesli oat flakes, Journal of Food Science, 67, 455-460.

doi:10.1111/j.1365-2621.2002.tb11428.x

[7] Kobus, J. and Flaczyk, E. (2007) Comparsion of antioxidant activity water extracts from leaves Morus alba $L$. and Ginkgo biloba L., Polish Journal of Human Nutrition and Metabolism, 34, 1382-1386.

[8] Kobus. J., Flaczyk, E., Siger, A.,Nogala-Kałucka, M., Korczak, J. and Pegg, R.B. (2009) Phenolic compounds and antioxidant activity of extracts of Ginkgo leaves. European Journal of Lipid Science and Technology, 111, 1150-1160. doi:10.1002/ejlt.200800299

[9] Klensporf-Pawlik, D. and Jeleń, H.H., (2009) Nitrogen atmosphere and natural antioxidants effect on muesli oxidation during long-time storage. Acta Scientarum Polonorum Technologia Alimentqria, 8, 5-15.

[10] Ercisli, S. and Orhan, E. (2007) Chemical composition of white ( Morus alba), red ( Morus rubra) and black ( Morus nigra) mulberry fruits. Food Chemistry, 103, 1380-1384. doi:10.1016/j.foodchem.2006.10.054

[11] Chon, S.-U., Kim, Y-M, Park, Y.-J., Heo, B.-G., Park, Y.-S. and Gorinstein, S. (2009) Antioxidant and antiproliferative effects of methanol extracts from raw and fermented parts of mulberry plant (Morus alba L.), European Food Research and Technology, 230, 231-237. doi:10.1007/s00217-009-1165-2

[12] Gundogdu, M., Muradoglu, F. Gazioglu Sensoyb R.I. and Yilmazc H. (2011) Determination of Fruit Chemical Properties of Morus nigra L., Morus alba L. and Morus rubra L. by HPLC. Scientia Horticulturae, 132, 37-41. doi:10.1016/j.scienta.2011.09.035

[13] AOAC (1984) Officials methods of analysis. 14th edition USA: Association of Official Analytical Chemist, Arlington, VA.
[14] PN-EN ISO 5508: 1996, Animal and vegetable oils and fats. The analysis of fatty acid methyl esters by gas chromatography.

[15] Cheung, L. M., Cheung, P. C. K. and Ooi, V. E. C. (2003) Antioxidant activity and total phenolcs of edible mushrooms extracts. Food Chemistry, 81, 249-255. doi.org/10.1016/S0308-8146(02)00419-3

[16] Amarowicz, R., Naczk, M. and Shahidi, F. (2000) Antioxidant activity of crude tannins of canola and rapeseed hulls. Journal of the American Oil Chemistry Society, 77, 957-961. doi:10.1007/s11746-000-0151-0

[17] Re, R., Pellegrini, N., Proteggente, A., Pannala, A., Yang, M. and Rice-Evans, C. (1999) Antioxidant activity applying in improved ABTS radical cation decolorization assy. Free Radical Biology and Medicine, 26, 1231-1237. doi:10.1016/S0891-5849(98)00315-3

[18] Flaczyk, E. Rudzińska, M. Wąsowicz, E. Korczak and J. Amarowicz R. (2006), Effect of cracklings hydrolysates on oxidative stability of pork meatballs fat. Food Research International, 39, 924-931. doi:10.1016/j.foodres.2006.05.004

[19] PN-ISO 3960: 2005. Animal and vegetable oils and fats Determination of peroxide value.

[20] PN-ISO 6885: 2008. Animal and vegetable oils and fats Determination of anisidine value

[21] PN-ISO 8589:1998, Sensory analysis - General guidance for laboratory of sensory analysis.

[22] Bae, S. H., and Suh, H. J. (2007) Antioxidant activities of five different mulberry cultivars in Korea. LWT - Food Science and Technology, 40, 955-962.

[23] Pawlowska,A.M. and Oleszek, W. and Braca, A. (2008) Quali-quantitative analyses of flavonoids of Morus nigra L. and Morus alba L. (Moraceae) fruits. Journal of Agricultural and Food Chemistry, 56, 3377-3380. doi:10.1021/jf703709r 